\title{
A Two-Level Desired Load Profile Tracking Algorithm for Electric Two-Wheeler Charging Stations
}

\author{
Duc Nguyen Huu \\ Faculty of Energy Technology \\ Electric Power University \\ Hanoi, Vietnam \\ ducnh@epu.edu.vn
}

\author{
Van Nguyen Ngoc \\ Faculty of Electrical Engineering \\ Electric Power University \\ Hanoi, Vietnam \\ vannn@epu.edu.vn
}

\begin{abstract}
In Vietnam and in other developing countries, twowheeled electric vehicles are potential alternatives to gasolinepowered motorbikes. The growth in the number of Electric TwoWheelers (E2Ws) requires a large power demand of charging load. In addition, the increasing spread in the appearance and penetration of rooftop photovoltaic (PV) power systems, with their intermittence and uncertain nature, poses technical challenges that need to be addressed. The coordination of PV rooftop operation and $\mathrm{EV}$ charging may be an effective solution to meet the emerging load demand from EVs, increasing solar power penetration while minimizing the cost of grid reinforcement or possible upgrades. In this paper, a two-level desired load profile tracking algorithm for PV integrated electric bicycles/electric motorcycle charging stations is proposed with the purposes of load leveling, valley filling, and peak shaving. The simulation results show that the proposed algorithm is an effective solution, significantly improving the load profile, especially when compared with uncontrolled charging and constant charging power scheme.
\end{abstract}

Keywords-electric two-wheelers; charging stations; load leveling; charging algorithm; solar power

\section{INTRODUCTION}

The transport sector currently accounts for about $20 \%$ of $\mathrm{CO}_{2}$ emissions and more than $50 \%$ of fossil fuel consumption. Recently, transport electrification has been considered as an effective measure to reduce Greenhouse Gas (GHG) emissions. However, basically, the GHG reduction purpose is only obtainable if the energy used for transport electrification is from renewable sources, instead of fossil fuel power plants [1]. Therefore, EV charging combined with renewable energy offers the potential for eco-friendly and sustainable development in both transportation and energy sectors. In Vietnam, the transition to electric mobility has notable characteristics. Instead of electric cars, electric bikes and motorcycles (hereafter referred to as electric two-wheelers or E2Ws) have been more attractive $[2,3]$. A continuous growing in the number of affordable E2Ws in urban traffic initiates necessities like charging points at homes and workplaces.
More charging load leads to a higher demand for electrical energy. With modest battery capacity and charging power compared to electric cars, E2Ws charging at home might utilize portable chargers (which came with EVs) plugging in standard socket outlets. Because of modest power rating, these chargers, often of one direction type, are designed to optimally supply electricity to the EV's battery without caring of the electricity price scheme and the impacts to the grid or to other loads. However, in locations like offices, supermarkets, campuses, apartment buildings, transport terminals, or public parkings, a high number of E2Ws charging simultaneously would introduce a very high charging demand which might impact the grid or the provided power quality. In such cases, dedicated charging facilities are required, which could regulate and coordinate charging loads in the station, decide each EV's charging pattern based on EV owners' settings, technical constraints and/or economic/technical objectives. In other business models, such as battery swapping stations, coordination charging of batteries in the station might be more imperative since it can help both station operators/service providers and $\mathrm{EV}$ users to maximize their benefits.

Regarding to the electrical energy sector, Vietnam has a great potential of solar power [4] exploitation. According to the draft national power development plan for the period 20212030, with a vision to 2045 (Draft Master Plan VIII or PDP8), the total installed capacity in 2020 was about $69,094 \mathrm{MW}$, with hydropower accounting for $30 \%$, coal-fired power for $30 \%$, gas natural/diesel power for $13 \%$, and renewable energy for $26 \%$ [5] of total production. Total solar power generation in 2020 was $10.6 \times 10^{9} \mathrm{kWh}$, of which rooftop solar power was $1.16 \times 10^{9} \mathrm{kWh}$. In comparison with other power sources, renewable energy, especially solar power, is developed very quickly in Vietnam. However, because of its intermittent and uncertain nature, the continuing growth in the share of PV power leads to grid issues such as power, voltage and frequency fluctuations. This requires expensive spinning reserves, directly affecting the economic efficiency. In addition, transmission/distribution system infrastructure investment does not keep up with PV development, which results in cut-downs in the solar output. 
By the end of December 31, 2020, there were 101,029 rooftop PV projects (nearly 9,296MWp installed capacity) connected to the national power system [5]. The development in the distributed rooftop solar power sources, along with the increasing popularity of E2 Ws, poses technical challenges that need to be addressed. Uncontrolled EV charging may increase the peak load demand and energy losses in residential buildings and distribution power grids [6-8], which result in inefficient operation of the system. Noteworthily, the higher amount of solar PV output usually corresponds to low load time (about from $10 \mathrm{AM}$ to $2 \mathrm{PM}$ ), resulting in needs of load shifting or energy storage systems. If the EV charging and discharging processes are properly controlled, EV batteries can also act as both loads and as energy storage devices or power regulating components. The involvement of EVs might contribute to increase renewable energy penetration without impact on grid stability because solar power is locally generated and consumed. The participation of EV batteries and PV systems contributes to smart grids with lots of potential such as load shifting, valley filling, peak clipping (peak shaving), regulation and spinning reserve. Several researches on optimal allocation of EVs are mentioned in [9], combined PV and EV charging in [10] and efficiency improvement of EV's motors in [11].

\section{A. Related Work}

It is shown that, the uncoordinated EV charging can trigger power loss and voltage deviation significantly [7]. With regard to load shifting and valley filling, studies focused on shifting the charging load to off-peak hours rather than uncontrolled charging. In [12], the authors proposed a decentralized valley filling strategy to build a day-ahead pricing scheme by solving the overall generation cost optimization problem (including base load and EVs). The pricing scheme is then broadcasted to vehicle owners to indirectly regulate charging behavior. Changing the charging pattern of each EV leads to valley filling at the system level. Authors in [13] proposed a decentralized charging algorithm for a large number of EVs by applying the Nash equilibrium principle. The authors found out that if the EVs are uniformly distributed, charging based on the Nash equilibrium principle would fill the overnight valley optimally. Linear programming was used in [14] to minimize the total electricity cost and reduce the power peak in a Time Of Use (TOU) price market while Quadratic Programming (QP) is utilized to minimize the overall load variance in a regional micro-grid in [15]. A decentralized charging protocol based on QP is also adopted to achieve the valley filling for the grid operators in [16]. In [17], a heuristic charging strategy for commercial buildings containing PV systems and EVs is proposed, where the EV charging rate is adjusted according to the variation of PV generation and the charging priority of each EV. Similarly, in [18], a centralized heuristic charging strategy is proposed to achieve the valley-filling for large scale EVs.

There are a very few studies relating E2Ws charging. Authors in [19] developed a PV-powered electric bicycle charging station providing AC/DC and wireless charging. The charging station had a built-in energy storage device that allows both grid-connected and standalone operations. Authors in [20] introduced a charging station powered by the grid, fuel cells, and PV to unburden the grid from charging. The station can provide service when power outages occur. However, the aforementioned studies were restricted to only developing a PV integrated charging station for E2Ws. They did not focus on E2Ws optimal charging especially when a large number of vehicles are charging simultaneously. In general, researchers mainly focus on charging algorithms for electric cars with large battery capacity and significant charging/discharging power. There are very few studies aimed at developing control algorithms and operation schemes for electric bicycles/electric motorbikes charging stations, although the potential of this vehicle type is undeniable in urban traffic. Besides, due to the great number of vehicles in the station, the application of multitarget optimal charging algorithms for electric cars may not be computationally feasible for E2Ws. A higher vehicle number in the station means a higher number of variables, which requires a higher computational capability.

\section{B. The Proposed Algorithm}

In this study, we propose a two-level desired load profile tracking algorithm to allocate charging power to electric bicycles/electric motorcycles. The algorithm has a small number of iterations and does not require high computational capability. The contributions of the proposed algorithm are summarized as follows:

The study proposes and develops an algorithm (level 1) to find out the total charging power scheme for E2Ws in the charging station. The total charging scheme and the netload compose the desired total load profile. Based on the total charging scheme, a power allocation algorithm (level 2) is proposed and utilized to decide the charging pattern of each E2W or group of E2Ws having similar parameters. These charging patterns satisfy the charging requirements of E2Ws and the aggregated charging powers approximate to the total charging power attained after level the implementation. The proposed algorithm is applied for load leveling, peak clipping, and valley filling. By utilizing the algorithm, rooftop PV penetration rate can be increased with lesser impact on microgrids. The algorithm helps to increase the penetration of E2Ws without requiring grid upgradation or reinforcement.

\section{SYSTEM MODELLING}

\section{A. EV Charging Infrastructure Model}

In this study, the charging station is controlled and managed by a centralized controller that optimizes the coordination of E2W loads with other loads and with the rooftop PV generation in order to improve load profile and perform valley filling and peak clipping. Based on the existing load, the controller estimates the total charging power pattern supplied to all E2Ws in the station. The estimation process is planed considering the total required energy of E2Ws and other technical constraints. The controller then allocates the total charging power to each E2W or a group of E2Ws at each period, which results in a specific charging pattern for each E2W or group. Assuming that the charging station accommodates $N$ electric bikes/electric motorcycles, $E V_{i}$ is the $i^{\text {th }} \mathrm{E} 2 \mathrm{~W}$ in the $N$-vehicle parking at the charging station. Let $C_{S}(t)$ be the total amount of energy stored in the batteries at time $t, D_{S}(t)$ is the non-EV load demand minus the power generated by solar panels at time $t$, also known as the netload, 
$E_{S}(t)$ is the total load demand (charging load plus netload); $T_{h}$ is the discretization in time, and $P_{S}(t)$ is the total charging power of all E2Ws at time $t$. We assume that the charging station is connected to the grid, to the load of the building, and to a rooftop PV system, and all chargers have a Vehicle to Grid (V2G) feature. Thus, the charging station can be described by the following discrete-time linear equations:

$$
\begin{gathered}
C_{S}(t+1)=C_{S}(t)+P_{S}(t) \cdot T_{h} \\
E_{S}(t)=D_{S}(t)+P_{S}(t)
\end{gathered}
$$

In this study, $T_{h}=0.5 \mathrm{~h}=30 \mathrm{~min}$. Since the chargers at the station include the V2G feature, the power exchange between the grid and E2Ws can be implemented in two directions. The charging behavior of all EVs can be determined by the sign of $E_{S}(t)$ where $E_{S}(t)>0\left(P_{S}(t)>0\right)$ if the aggregated batteries receive energy from the microgrid and $E_{S}(t)<0\left(P_{S}(t)<0\right)$ if the aggregated batteries discharge energy to the grid. The power constraints of charging and discharging processes (assuming all the EVs in the station are connected to the chargers) can be described as:

$$
\begin{gathered}
\text { Charging: } P_{S}(t) \leq \min \left\{\sum_{i=1}^{N} P_{\text {cmax }_{i}}, \sum_{i=1}^{N} P_{\text {bmax }_{i}}\right\} \\
\text { Discharging: } P_{S}(t) \geq \max \left\{\sum_{i=1}^{N} P_{c \min _{i}}, \sum_{i=1}^{N} P_{\text {bmin }_{i}}\right\}
\end{gathered}
$$

where $P_{c \text { max }_{i}}$ and $P_{c m i n}$ are the maximum charging and discharging power of charger $i$ respectively and $P_{\text {bmax }_{i}}, P_{b m i n}$ are the maximum permitted charging and discharging powers of $E V_{i}$.

It is assumed that all E2Ws have the same $P_{b \max }, P_{b \min }$ and $P_{c \max }, P_{c m i n}$ of all chargers are uniform. Then, the constraints in equations ( 3 ) and (4) become:

$$
\begin{gathered}
\text { Charging: } P_{S}(t) \leq \min \left\{N . P_{c \max }, N . P_{b \max }\right\} \\
\text { Discharging: } P_{S}(t) \geq \max \left\{N . P_{c \min }, N . P_{b \min }\right\}
\end{gathered}
$$

where $P_{c \min }$ (discharge) and $P_{c \max }$ (charge) are respectively the maximum permitted discharging/charging powers of the chargers. $P_{b \min }$ (discharge), $P_{b \max }$ (charge) are the maximum allowable discharging/charging powers of the battery, depending on the battery status. In this study, it is assumed that the charging power of an E2 W has a positive sign and has a highest value of $400 \mathrm{~W}$. Discharging power is negative and the maximum value is $-400 \mathrm{~W}$.

In addition, the total charging and discharging power must not exceed the feeder capacity:

$$
\begin{gathered}
\text { Charging: } P_{S}(t) \leq P_{\text {feedermax }}-D_{S}(t)=P_{\text {amax }} \\
\text { Discharging: } P_{S}(t) \geq-P_{\text {feedermax }}+D_{S}(t)=-P_{\text {amax }}
\end{gathered}
$$

where $P_{\text {amax }}$ is the maximum power that the grid can exchange with the EV loads, which is equivalent to the maximum gridmicrogrid exchange power $\left(P_{\text {feedermax }}\right)$ minus the net load. It is assumed that the non-EV load demand is uncontrolled. The charging station needs to control the total charging demand of the EVs so as to do not violate the constrains (7) and (8). The constraint on energy requirement of $\mathrm{E} 2 \mathrm{Ws}$ is:

$$
\begin{gathered}
R_{S}=\sum_{i=1}^{N} \text { Required Energy of EVi } \\
=\sum_{i=1}^{N} A_{i}\left(\text { LastSOC } C_{E V i}-\text { InitSOC }_{E V i}\right) \\
=\sum_{i=1}^{N}\left(\int_{t_{0}}^{t_{m}} P_{i}(t)\right)=1-p
\end{gathered}
$$

where $R_{S}$ is the total amount of the energy required by all the vehicles in the station, $A_{i}$ is $E V i$ 's battery capacity, $\operatorname{LastSOC}_{E V i}$ is the SOC of $E V i$ at the end of the working day, InitSOC $_{E V i}$ is the initial SOC of $E V i, t_{0}$ is the beginning time of the charging process, $t_{m}$ is the departure time, and $P_{i}(t)$ is the charging/discharging power of $E V i$ at time t. If a working day from time $t_{0}$ to the $t_{m}$ is divided into $M$ periods with duration $T_{h}$, the above constraint becomes:

$$
\begin{gathered}
R_{S}=\sum_{i=1}^{N} A_{i}\left(\text { LastSOC }_{E V i}-\text { InitSOC }_{E V i}\right) \\
=\sum_{i=1}^{N}\left(\sum_{j=1}^{M} P_{i j} \cdot T_{h}\right)
\end{gathered}
$$

where $P_{i j}$ is the charging power of $E V i$ at period (4).

Individually, each EV must satisfy the following constraints:

The amount of required energy constraint:

$$
\begin{gathered}
R_{E V i}=A_{i}\left(\text { LastSOC }_{E V i}-\text { InitSOC }_{E V i}\right) \\
=\int_{t_{0}}^{t_{m}} P_{i}(t)=\sum_{j=1}^{M} P_{i j} \cdot T_{h}
\end{gathered}
$$

The constraint on charging/discharging power:

$$
\text { Charging: } P_{i}(t) \leq \min \left\{P_{\text {cmax }}, P_{b \max }\right\}=\overline{P_{E V \iota}}
$$

$$
\text { Discharging: } P_{i}(t) \geq \max \left\{P_{c m i n}, P_{b m i n}\right\}=\underline{P_{E V i}}
$$

In this study, it is assumed that a charging station is built on the campus of EPU, servicing 160 electric bicycles/electric motorbikes during working hours from 7 AM to $17 \mathrm{PM}$. The E2W's battery has a capacity of $1200 \mathrm{Wh}$. Maximum charging/discharging powers are 400/-400W, respectively. Batteries are not allowed to discharge if the SOC is less than $10 \%$. At the end of the working day, the E2Ws' batteries are full.

\section{B. Rooftop PV Simulation}

We conduct this study using data from our previous work on the feasibility of PV-Integrated Charging Station for E2Ws [3]. Accordingly, the rooftop PV system is designed and simulated as follows:

- Capacity: $150 \mathrm{kWp}$.

- Location: Electric Power University (EPU), 21 02'49", 10547'07"; Hoang Quoc Viet Street, Cau Giay District, Vietnam.

- Scale: Most of electric bicycles / motorbikes currently adopt battery capacity of about $0.57-1.2 \mathrm{kWh}$. The charging station is assumed to serve about $160 \mathrm{E} 2 \mathrm{Ws}$ staying at the station from 7 h00 AM to 17 h00 PM.

- Sunshine hours, solar irradiation, temperature at the site are shown in Table I. 
TABLE I. SOLAR IRRADIATION PARAMETERS

\begin{tabular}{|c|c|c|}
\hline Parameter & Daily average value & Yearly average value \\
\hline $\begin{array}{c}\text { Direct normal irradiation } \\
\text { (DNI) }\end{array}$ & $1.688 \mathrm{kWh} / \mathrm{m}^{2} /$ day & $616 \mathrm{kWh} / \mathrm{m}^{2} /$ year \\
\hline $\begin{array}{c}\text { Global horizontal } \\
\text { irradiation (GHI) }\end{array}$ & $3.584 \mathrm{kWh} / \mathrm{m}^{2} /$ day & $1308 \mathrm{kWh} / \mathrm{m}^{2} /$ year \\
\hline $\begin{array}{c}\text { Diffuse horizontal } \\
\text { irradiation (DIF) }\end{array}$ & $2.320 \mathrm{kWh} / \mathrm{m}^{2} /$ day & $847 \mathrm{kWh} / \mathrm{m}^{2} /$ year \\
\hline $\begin{array}{c}\text { Global tilted irradiation at } \\
\text { optimum angle }\end{array}$ & $3.652 \mathrm{kWh} / \mathrm{m}^{2} /$ day & $1333 \mathrm{kWh} / \mathrm{m}^{2} /$ year \\
\hline Air temperature & \multicolumn{2}{|c|}{$24.1^{\circ} \mathrm{C}$} \\
\hline Terrain elevation & \multicolumn{2}{|c|}{$12 \mathrm{~m}$} \\
\hline
\end{tabular}

After conducting the simulation, the power output of the PV system in a typical day of each month was obtained and is shown in Table II.

\section{Non-EV Load Profile}

In order to meet the charging demands of E2Ws, the charging station at the EPU is interconnected with the grid and the rooftop PV system. It is also connected to building's loads, forming a microgrid. The load profile of non-EV loads in a typical day is shown in Figure 1. For the purpose of testing the algorithm effectiveness, we did not investigate non-EV load profiles in a typical working day/weekend and we did not account for load demand variation in different seasons. We assumed that the daily profile of non-EV loads is homogeneous. It is realized that a working day consists of two peaks: The first peak occurs at about 10 AM and the second at around 14 PM. The difference between the highest and the lowest load demand is quite large with the highest load being more than 3.6 times higher than the lowest. When considering rooftop PV power, the net load profiles in January and July are shown in Figures 2 and 3.

The integration of the $150 \mathrm{kWp}$ rooftop PV system significantly reduces electricity consumption, but the unmatched figures of solar power profile and non-EV load profile result in more serious variations in the netload. Off-peak periods of netload usually occur around 12 AM due to high solar power and low non-EV load. In January, the netload reaches the peak of nearly $40 \mathrm{~kW}$ at $8 \mathrm{AM}$. This figure is 5.7 times greater than the lowest value of $7 \mathrm{~kW}$ at $12 \mathrm{AM}$. In July, the netload is even negative from 10:305 AM to 14 PM, meaning that if there is no charging load participation, the rooftop PV power, after meeting load demand, can be injected into the grid.

\section{THE PROPOSED ALGORITHM}

To implement load leveling we propose a two-level desired load profile tracking algorithm.

\section{A. Level 1: Total Charging Pattern Finding}

This level includes the following steps:

- At the beginning of the process, collect the data of E2 Ws in the charging station.

- Collect the data of non-EV load and solar power output forecast.

- Calculate the total load demand and the total energy demand of E2Ws.

TABLE II. SOLAR POWER OUTPUT (W)

\begin{tabular}{|c|c|c|c|c|c|c|c|c|c|c|c|c|}
\hline & Jan & Feb & Mar & Apr & May & Jun & Jul & Aug & Sep & Oct & Nov & Dec \\
\hline $0-5$ & 0.0 & 0.0 & 0.0 & 0.0 & 0.0 & 0.0 & 0.0 & 0.0 & 0.0 & 0.0 & 0.0 & 0.0 \\
\hline $5-5.5$ & 0.0 & 0.0 & 0.0 & 0.0 & 307.7 & 307.7 & 333.3 & 0.0 & 0.0 & 0.0 & 0.0 & 0.0 \\
\hline 5.5-6 & 0.0 & 0.0 & 0.0 & 0.0 & 1692.3 & 1692.3 & 1666.7 & 0.0 & 0.0 & 0.0 & 0.0 & 0.0 \\
\hline 6-6.5 & 0.0 & 0.0 & 285.7 & 3125.0 & 7407.4 & 7407.4 & 6352.9 & 5446.8 & 3600.0 & 2777.8 & 695.7 & 307.7 \\
\hline $6.5-7$ & 0.0 & 0.0 & 1714.3 & 6875.0 & 12592.6 & 12592.6 & 11647.1 & 10553.2 & 8400.0 & 7222.2 & 3304.3 & 1692.3 \\
\hline $7-7.5$ & 4235.3 & 5953.5 & 8800.0 & 14450.0 & 20756.8 & 20571.4 & 20756.8 & 19592.6 & 18438.1 & 17465.3 & 13927.7 & 7692.3 \\
\hline $7.5-8$ & 7764.7 & 10046.5 & 13200.0 & 19550.0 & 27243.2 & 27428.6 & 27243.2 & 26407.4 & 25561.9 & 24534.7 & 20072.3 & 12307.7 \\
\hline $8-8.5$ & 14222.2 & 16790.7 & 19555.6 & 26488.2 & 36000.0 & 36571.4 & 35788.2 & 35788.2 & 36000.0 & 34795.2 & 29257.1 & 19168.3 \\
\hline 8.5-9 & 17777.8 & 21209.3 & 24444.4 & 31511.8 & 42000.0 & 43428.6 & 42211.8 & 42211.8 & 42000.0 & 41204.8 & 34742.9 & 24831.7 \\
\hline $9-9.5$ & 21942.9 & 26698.4 & 30461.5 & 37426.9 & 49614.7 & 52608.7 & 50841.6 & 50612.6 & 49843.3 & 49843.3 & 41634.4 & 32885.9 \\
\hline $9.5-10$ & 26057.1 & 31301.6 & 35538.5 & 42573.1 & 54385.3 & 57391.3 & 55158.4 & 55387.4 & 54156.7 & 54156.7 & 46365.6 & 37114.1 \\
\hline 10-10.5 & 31565.2 & 37097.6 & 42316.9 & 49308.1 & 60298.0 & 63533.8 & 61015.9 & 62015.6 & 59775.1 & 60259.1 & 52778.3 & 42549.5 \\
\hline 10.5-11 & 34434.8 & 40902.4 & 45683.1 & 52691.9 & 63702.0 & 66466.2 & 62984.1 & 63984.4 & 62224.9 & 61740.9 & 55221.7 & 45450.5 \\
\hline 11-11.5 & 37788.8 & 45782.4 & 50260.9 & 57016.9 & 68258.1 & 70750.9 & 65503.8 & 67000.0 & 65750.9 & 63751.0 & 58504.2 & 49261.1 \\
\hline 11.5-12 & 40211.2 & 48217.6 & 51739.1 & 58983.1 & 69741.9 & 71249.1 & 66496.2 & 67000.0 & 66249.1 & 64249.0 & 59495.8 & 50738.9 \\
\hline 12-12.5 & 44251.4 & 52504.9 & 55018.9 & 63275.7 & 73014.1 & 73793.6 & 69014.9 & 68796.9 & 68534.4 & 67063.5 & 62276.2 & 54019.2 \\
\hline 12.5-13 & 43748.6 & 51495.1 & 52981.1 & 60724.3 & 70985.9 & 70206.4 & 66985.1 & 65203.1 & 65465.6 & 62936.5 & 59723.8 & 51980.8 \\
\hline 13-13.5 & 45097.6 & 52083.3 & 52356.0 & 59342.5 & 70595.4 & 67871.5 & 66601.6 & 62608.7 & 64155.2 & 60729.0 & 59450.2 & 51634.4 \\
\hline 13.5-14 & 40902.4 & 47916.7 & 47644.0 & 54657.5 & 65404.6 & 62128.5 & 61398.4 & 57391.3 & 57844.8 & 53271.0 & 52549.8 & 46365.6 \\
\hline 14-14.5 & 38281.3 & 45522.6 & 45127.5 & 52067.8 & 62296.3 & 57742.6 & 57742.6 & 53763.4 & 53355.6 & 47410.3 & 47716.1 & 43457.1 \\
\hline 14.5-15 & 31718.8 & 38477.4 & 36872.5 & 43932.2 & 53703.7 & 50257.4 & 50257.4 & 46236.6 & 44644.4 & 38589.7 & 38283.9 & 34542.9 \\
\hline $15-15.5$ & 26784.8 & 33306.9 & 29714.3 & 37551.7 & 47040.0 & 44444.4 & 44137.9 & 39876.9 & 37551.7 & 32044.0 & 31441.9 & 27842.1 \\
\hline 15.5-16 & 19215.2 & 24693.1 & 22285.7 & 28448.3 & 36960.0 & 35555.6 & 35862.1 & 32123.1 & 28448.3 & 21956.0 & 20558.1 & 18157.9 \\
\hline 16-16.5 & 12903.2 & 17818.2 & 16095.2 & 21018.2 & 28800.0 & 28444.4 & 29411.8 & 26520.5 & 21407.4 & 12945.0 & 10364.4 & 9074.1 \\
\hline 16.5-17 & 7096.8 & 10181.8 & 9904.8 & 12981.8 & 19200.0 & 19555.6 & 20588.2 & 17479.5 & 12592.6 & 7055.0 & 5635.6 & 4925.9 \\
\hline $17-17.5$ & 1333.3 & 2666.7 & 4000.0 & 5333.3 & 10666.7 & 11571.4 & 12903.2 & 9333.3 & 4000.0 & 1200.0 & 933.3 & 800.0 \\
\hline 17.5-18 & 666.7 & 1333.3 & 2000.0 & 2666.7 & 5333.3 & 6428.6 & 7096.8 & 4666.7 & 2000.0 & 600.0 & 466.7 & 400.0 \\
\hline 18-18.5 & 0.0 & 0.0 & 0.0 & 0.0 & 0.0 & 2000.0 & 2000.0 & 0.0 & 0.0 & 0.0 & 0.0 & 0.0 \\
\hline 18.5-24 & 0.0 & 0.0 & 0.0 & 0.0 & 0.0 & 0.0 & 0.0 & 0.0 & 0.0 & 0.0 & 0.0 & 0.0 \\
\hline
\end{tabular}




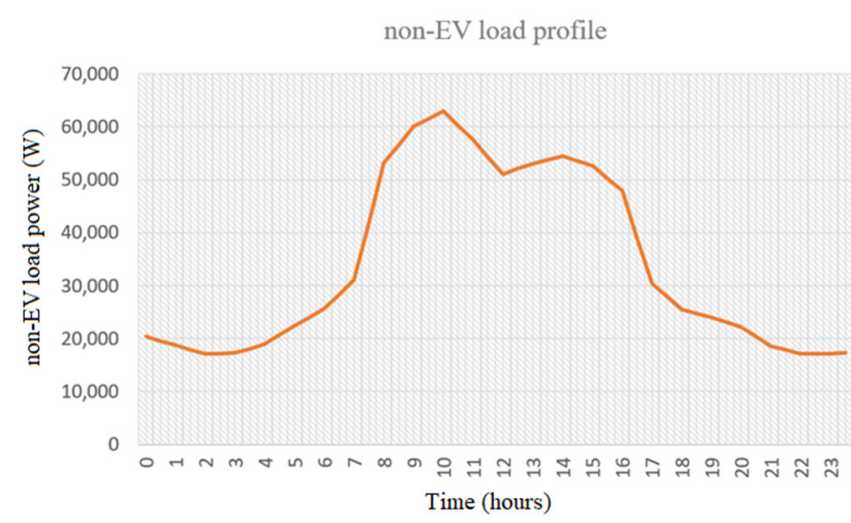

Fig. 1. Non-EV load profile.

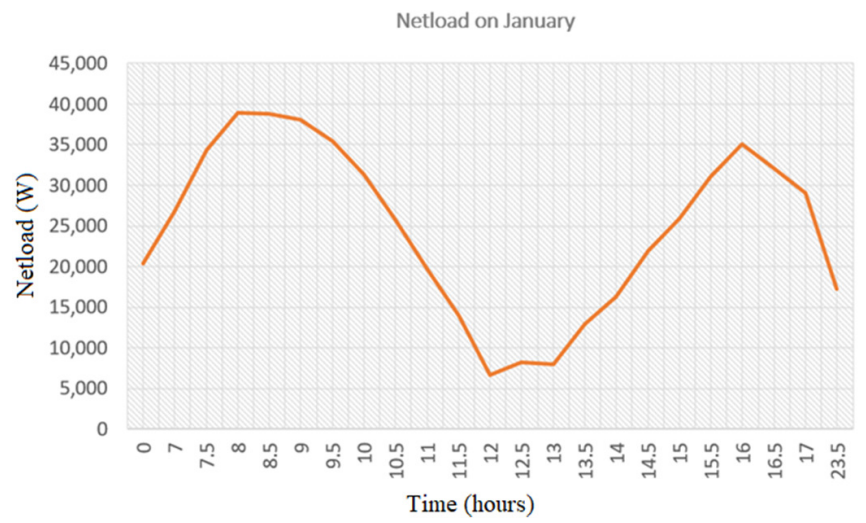

Fig. 2. Netload in January.

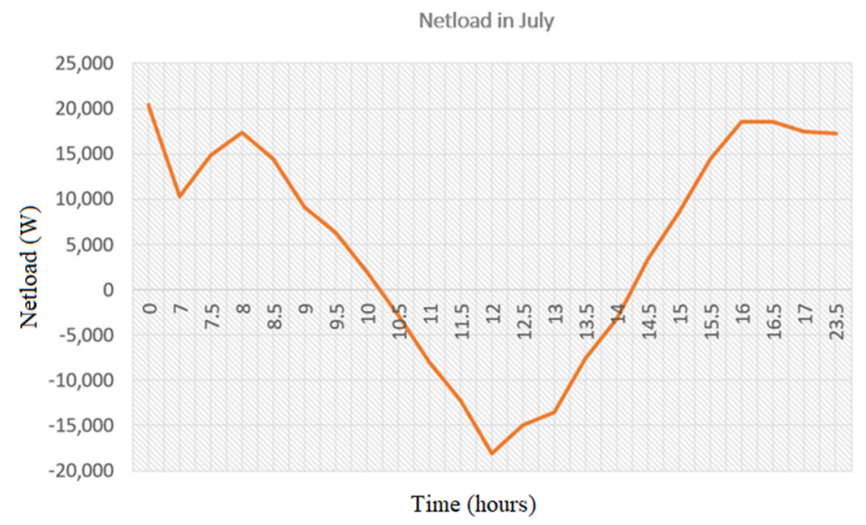

Fig. 3. Netload in June.

- Define the desired total load profile (including charging load and netload) which satisfies the total load demand as well as the charging energy requirement.

- At each period, preliminary total charging power can be obtained by subtracting the netload from the desired total load.

- Browse all periods, implement correction if at a certain period the total charging power of E2Ws violates the constraints. Reallocate the power at the unsatisfactory period for the remaining periods in order to ensure that at the end of the working day, the total amount of energy supplied to the E2Ws meets the requirements. At the end of this step, we obtain an improved total charging power profile.

- Calculate the battery capacity of the representative. Browse all periods, check the Depth Of Discharge (DOD) and the maximum battery capacity constraints. Reallocate charging power at the unsatisfactory period for the remaining time steps. At the end of this reallocation, we find the final total charging pattern.

\section{B. Level 2}

Based on the total charging pattern obtained at Level 1, allocating the total charging power for each EV or groups of similar parameter EVs is conducted. Level 2 includes the following steps:

- Browse the EV groups.

- Repeat the steps of Level 1 in order to identify the preliminary charging power pattern for the considered group.

- Check the charging/discharging power constraints at each period.

- Browse all periods, conduct corrections and reallocate if there is any charging/discharging power constraint violation.

- Calculate battery capacity. Check whether there is any DOD or maximum battery capacity violation. If the constraints are violated, reallocate the charging/discharging power of the unsatisfactory period for the remaining periods.

- After the two corrections above, conduct the third reallocation, so as to the sum of EV groups' charging power at each period reaches the total charging demand (after Level 1) with predefined error.

The pseudocode of the proposed algorithm is shown below.

C. Algorithm: Desired TotalLload Profile Tracking-Load Leveling

1: INPUT: At the beginning, the controller acquires data of E2Ws in the charging station. The data includes:

- The battery capacity of $E V_{i}: A_{i}$

- The initial SOC and the required SOC at the departure time of the $E V_{i}:$ InitSOC $_{E V i}$; LastSOC LVi $_{1}$

- The maximum charging and discharging power of $E V_{i}: \overline{P_{E V l}}$ (charging); $P_{E V i}$ (discharging)

- The permitted depth of discharge of $E V_{i}: D O D_{E V i}$

- Besides, non-EV load forecast and PV power output forecast are also collected.

2: OUTPUT: At each time step $t=1,2,3, \ldots, M$, output the total desired charging power.

\section{3: PROCEDURE:}


4: Total required energy assignment: $R_{S} \leftarrow 0$

5: for $i=1$ to $N$ do

6: Calculate the required energy of $E V_{i}$ :

$$
R_{E V i} \leftarrow A_{i}\left(\text { LastSOC }_{E V i}-\text { InitSOC }_{E V i}\right)
$$

7: $\quad R_{S}:=R_{S}+R_{E V i}$

8: end for

9: Calculate the initial SOC of an EV which can be a representative of $\mathrm{E} 2 \mathrm{Ws}$ in the station.

10: Preliminary allocation of $R_{S}$ for $M$ periods. Calculate the preliminary total charging power at each time step:

$$
P_{\text {pre } \_} \leftarrow \frac{R_{S}}{M \cdot T_{h}}
$$

11: for $i=1$ to $M$ do

12:Calculate netload at each time step:

$$
D_{i}=P_{n o n-E V_{-} i}-P_{\text {solar } \_} i
$$

13: Calculate the total load: $E_{S_{-} i}=D_{S_{-} i}+P_{\text {pre }} S$

14: end for

15: Average $E_{S_{i} i}$ in $M$ time periods. From the desired load profile, get the total load demand at each time step $E_{S D_{-} i}$

16: for $i=1$ to $M$ do

17: Calculate the preliminary total charging power at each time step: $P_{S i}=E_{S D_{-} i}-D_{i}$

18: Check charging/discharging power constrains at period $i$ $\left(P_{S i}\right)$

19: if $P_{S i}$ violates power constraints

20: Reallocate $P_{S i}$ for the remaining periods in order to ensure that at the end of the working day, the total amount of the energy supplied to the E2Ws meets the requirements and the allocation does not make charging powers at the remaining periods violate the power constraints.

21 :end if

22: end for

23: for $i=1$ to $M$ do

24: Calculate battery capacity of the representative at period $i$.

25: if the battery capacity at period $i$ violates DOD or maximum battery capacity constraints

26: Reallocate the charging power at the period (which violates the constraints) for the remaining periods. The allocation does not make charging powers at the remaining periods violate the power constraints.

27: end if

28: end for

\section{SimUlATION AND ANALYSIS}

The current study assumes that the accurate day-ahead forecast of non-EV load and generation capacity forecast of the solar power system are available. For the purpose of verifying the effectiveness of the proposed algorithm, we did not consider the variations in the accuracy of the forecasts. With 160 E2 Ws in the charging station, we relied on the research data of [21] about the charging behavior. Accordingly, the initial SOC distribution is shown in Table III.

TABLE III. INITIAL SOC DISTRIBUTION

\begin{tabular}{|c|c|c|}
\hline Percentage & Initial SOC & Group \\
\hline $11.6 \%$ & $90-100 \%$ SOC & G1 \\
\hline $14.8 \%$ & $80-90 \%$ SOC & G2 \\
\hline $14.9 \%$ & $70-80 \%$ SOC & G3 \\
\hline $14.9 \%$ & $60-70 \%$ SOC & G4 \\
\hline $13.7 \%$ & $50-60 \%$ SOC & G5 \\
\hline $10.8 \%$ & $40-50 \%$ SOC & G6 \\
\hline $8.4 \%$ & $30-40 \%$ SOC & G7 \\
\hline $6.1 \%$ & $20-30 \%$ SOC & G8 \\
\hline $3.5 \%$ & $10-20 \%$ SOC & G9 \\
\hline $1.3 \%$ & $0-10 \%$ SOC & G10 \\
\hline
\end{tabular}

The average initial state of charge of the EVs is $62 \%$. In more than two thirds of all charges, the initial state of charge was above $50 \%$ SOC and $11.6 \%$ of all charges were even initiated with a nearly full battery with more than $90 \%$ SOC. The required amount of energy $\left(R_{S}\right)$ of the $160 \mathrm{E} 2 \mathrm{Ws}$ is determined by (9). The battery capacity of an E2W is $1200 \mathrm{~W}$ and the maximum allowable charging/discharging power is 400/-400W respectively. In this study, we divided the 160 EVs into 10 groups as shown in Table III. In order to investigate the efficiency of the algorithm, simulations were conducted considering three cases:

- Case 1: Max speed charging: E2Ws are charged at maximum charging rate from 7 AM until reaching the required amount of energy.

- Case 2: Constant charging power: E2Ws are charged at a constant rate during working time so that the required amount of energy is attainable at $17 \mathrm{PM}$.

- Case 3: Smart charging: E2Ws follow the proposed algorithm.

The simulation results of the three cases during January, which is the month having the lowest solar power generation, are shown in Figure 4. It was found that, in case 1, when the E2Ws are charged at the maximum rate, the peak load occurred at 7:30 AM. This is 14.7 times higher than lowest load at 12 $\mathrm{AM}$ and 2.5 times higher than the netload peak value. When the vehicles are charged at constant power, the difference between peak and base load does not improve compared to the netload profile. The peak load, which occurs at $8 \mathrm{AM}$, is $46.25 \mathrm{~kW}$ and the base load is $14 \mathrm{~kW}$ at $12 \mathrm{AM}$. With the proposed algorithm, the charging and discharging powers of the vehicles are controlled to reduce peak load and perform valley filling and load leveling. The load power from $7 \mathrm{AM}$ to $17 \mathrm{PM}$ ranges from $32.27 \mathrm{~kW}$ to $33.53 \mathrm{~kW}$. At the last time steps, although the netload is higher than the average load, the E2Ws' battery does not discharge to ensure that it is full at the 
departure time. In case 3, the profile of total charging load and charging profiles of typical E2Ws in 10 groups are shown in Figures 5 and 6.

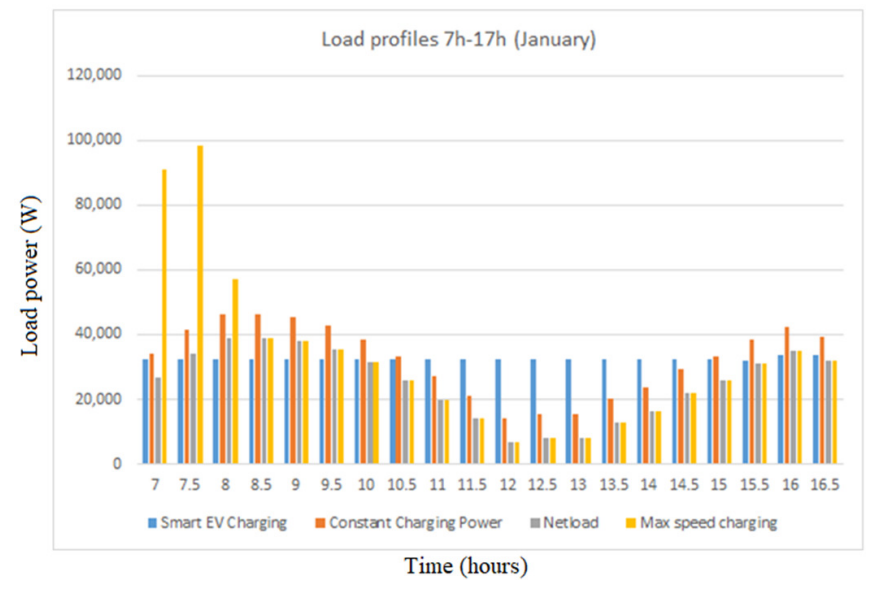

Fig. 4. Load profile in January.

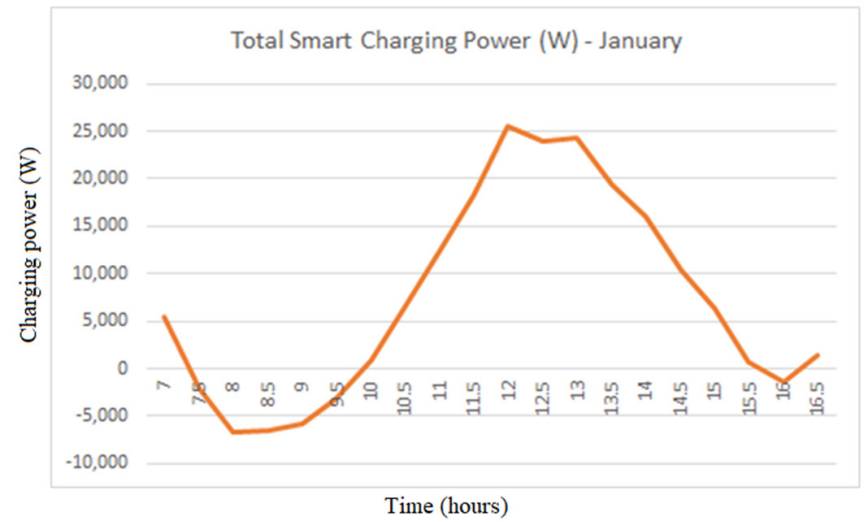

Fig. 5. Total charging load profile in case 3 .

Typical charging patterns of $10 \mathrm{EV}$ groups - January

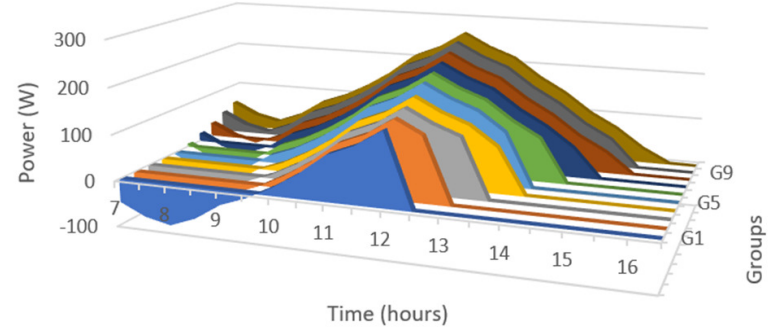

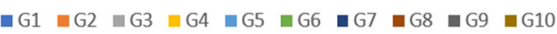

Fig. 6. Charging profiles of the $10 \mathrm{E} 2 \mathrm{~W}$ groups in case 3 .

Generally, it is found that, from 7:30 AM to $10 \mathrm{AM}$, the total charging power of E2Ws in the station is negative, meaning that the batteries discharge energy, partly powering non-EV loads so as to reduce the peak load. At the hours of large solar power and low netload, the total charging power increases in order to absorb electricity from the solar panels and perform valley filling. Considering the typical charging patterns of the $10 \mathrm{E} 2 \mathrm{~W}$ groups, group 1 which has the biggest initial SOC, performs energy discharge during the first hours, whereas group 10 has the lowest Initial SOC. So, in general, this group is mainly charged from $7 \mathrm{AM}$ to $17 \mathrm{PM}$. The change in the battery capacity representing the $160 \mathrm{E} 2 \mathrm{Ws}$ in the station is shown in Figure 7. The battery capacity increases rapidly from 10:30 AM and 15 PM, corresponding to the periods of highest solar power and charging rate. Figure 8 illustrates the typical battery capacity variation of the $10 \mathrm{E} 2 \mathrm{~W}$ groups.

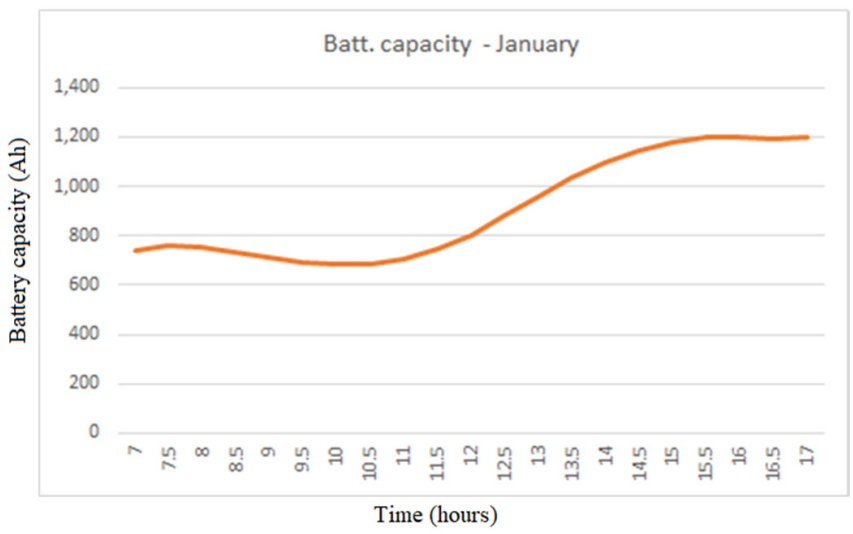

Fig. 7. Battery capacity profile of the representative in January.

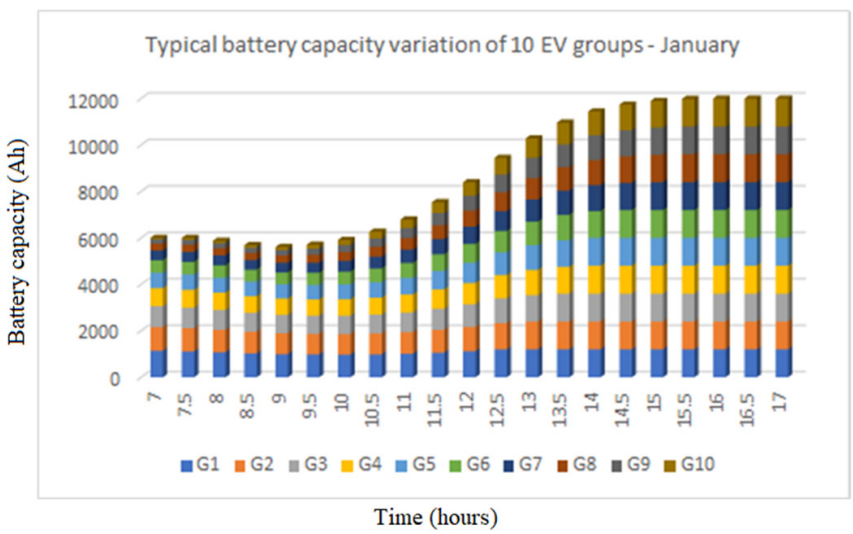

Fig. 8. Typical battery capacity variation of $10 \mathrm{E} 2 \mathrm{~W}$ groups in January.

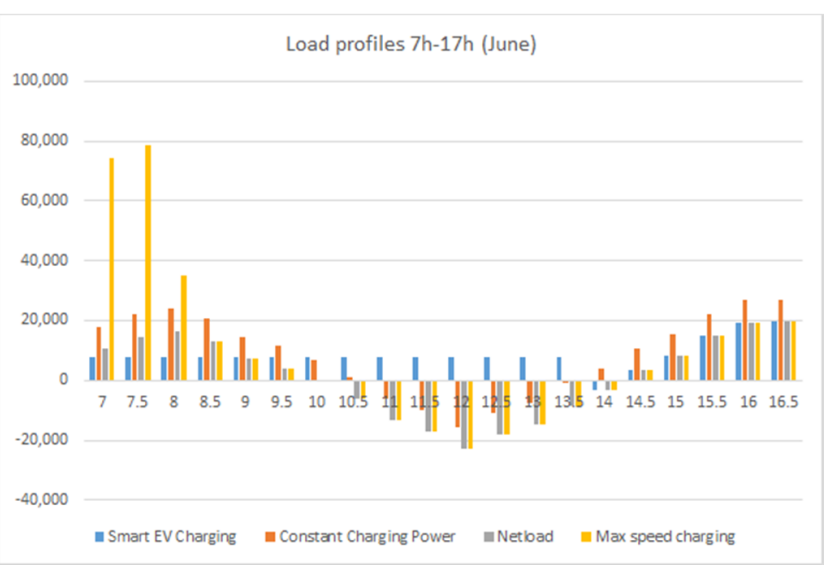

Fig. 9. Load profile in June. 
The load profile for the three different charging schemes during June (the month with the largest solar power generation) is shown in Figure 9. Compared to January, the netload in June is much lower because this is the month with the largest solar power output. If there is no charging load or even there is a charging load following scheme 1 or 2 , at high solar power output duration, PV generation does not only meet the load demand but also it can inject the surplus power into the grid. However, in case 1, the simultaneous charging of E2Ws as soon as they are connected to the chargers makes the total load reach a very high peak, up to $78.67 \mathrm{~kW}$ at $7: 30 \mathrm{AM}$. In case 2 , the peak load reaches $26.9 \mathrm{~kW}$ at $16: 30 \mathrm{PM}$, which is significantly lower than the peak load in case 1 . In the case of charging following the proposed algorithm, the peak load decrease does not improve compared to case 2 , but the load fluctuation is significantly reduced. The valley filling effect is clearly observable from 10:30 AM to 13:30 PM and peak shaving occurs from 7 AM to 9:30 AM. The total charging power profile during June and the charging profiles of the 10 E2W groups are shown in Figures 10 and 11. Generally, with the high solar power in June, E2Ws' batteries only discharge energy to reduce peak load in the early morning. From 9 AM onwards, when the solar power output is high, the battery will be in the charging process. In comparison to January, the charging ends earlier as the excess solar power allows the vehicles to be charged at a higher power.

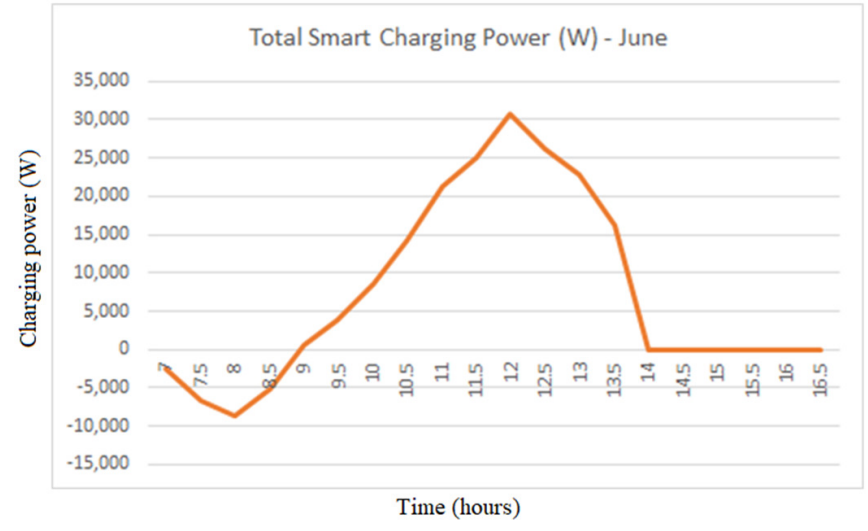

Fig. 10. Total charging power in June.

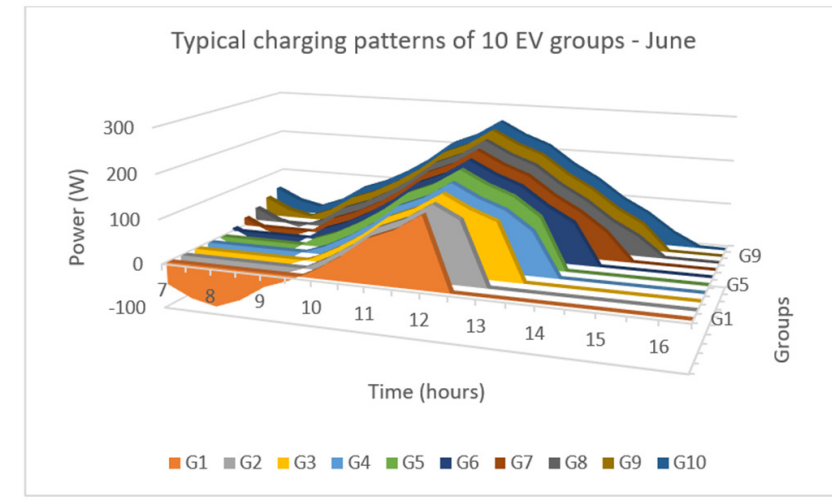

Fig. 11. Charging patterns of the $10 \mathrm{E} 2 \mathrm{~W}$ groups.

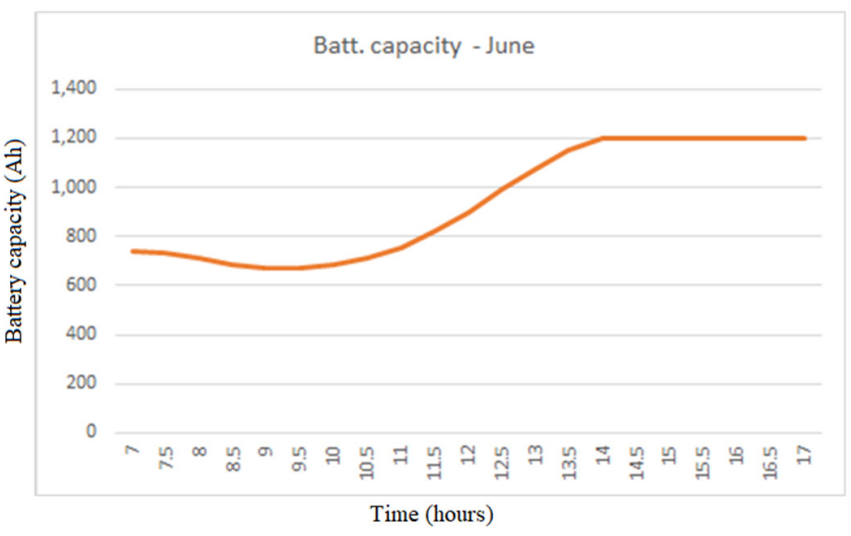

Fig. 12. Battery capacity profile of the representative during June.

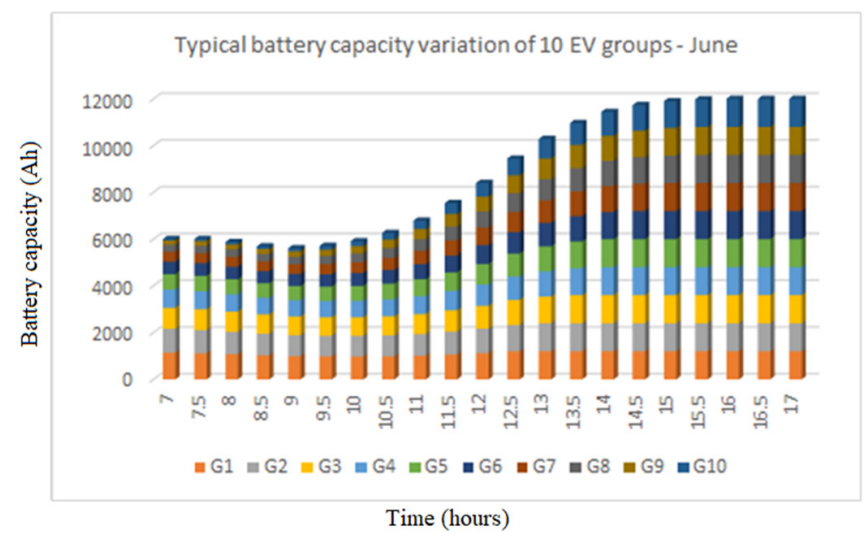

Fig. 13. Typical battery capacity variation of the $10 \mathrm{E} 2 \mathrm{~W}$ groups during June.

The battery capacity change of the representative is shown in Figure 12. Figure 13 shows the battery capacity variation of the $10 \mathrm{E} 2 \mathrm{~W}$ groups. By about $14 \mathrm{PM}$, most E2Ws were fully charged. At the end of the working day, the batteries were not discharged to ensure that the vehicles are fully charged at 17 PM.

\section{CONCLUSIONS}

In case of a given electricity tariff, optimizing EV charging cost may result in a large number of vehicles charging simultaneously during low-cost price periods. This can lead to unwanted peak load and an adversely impact on the grid stability and power quality. If the utilities adjust electricity tariff according to real-time load demand variations (indirectly adjusting to the charging behavior of EV owners), the algorithm that identifies real-time tariff may not converge.

Unlike the case of electric cars, the decentralized approaches through adjusting the electricity tariff do not make much sense in changing the behavior of individual E2W owner because the charging power and battery capacity of E2Ws are much lower. However, when a large number of electric bicycles and electric scooters are present in buildings, offices, campuses, public parking, etc., the uncontrolled charging of that large number of E2Ws at the same time might trigger many unwanted technical problems. 
Compared to other studies on load leveling using centralized approach, the proposed algorithm does not focus on forming and solving optimal objective functions. Instead, we derive from the estimated total amount of energy required by EVs and non-EV loads to establish the desired total load profile. Charging power is then allocated to the E2Ws on the basis of that desired profile and relevant constraints. Therefore, when compared to prior studies [15, 16, 22-24] our algorithm does not require high computational capability or computational time of the controller for solving optimization problems. It also ensures the finding each E2W's or group of E2Ws' charging schedule. This is especially meaningful because the number of E2Ws in the charging station can be very higher than the number of electric cars, leading to a higher number of variables in the optimization problems.

In this study, we propose a power distribution algorithm for E2Ws in an integrated PV charging station. The controller implements a two-level algorithm tracking the desired load profile in order to perform load leveling, valley filling, and peak clipping. The study offers a novel approach to control and coordinate E2W charging loads in the integrated PV charging station in which the controller decides the charging pattern for each E2W to improve the overall load profile. Simulation results show that, when compared to uncontrolled charging and constant power charging scheme, the proposed algorithm proves its effectiveness in load leveling, dramatically narrowing the load fluctuations while the peak load is even lower than the highest value of netload. The proposed algorithm can help increasing the penetration rate of both E2Ws and PV power and can indirectly lighten the adverse impacts on the microgrid and reduce the need of grid upgradation or reinforcement. In our future studies, we will further carry out research on the optimal control methods based on the available energy of distributed-mobile storage EVs as in [25-28].

\section{REFERENCES}

[1] M. Messagie, F.-S. Boureima, T. Coosemans, C. Macharis, and J. V. Mierlo, "A Range-Based Vehicle Life Cycle Assessment Incorporating Variability in the Environmental Assessment of Different Vehicle Technologies and Fuels," Energies, vol. 7, no. 3, pp. 1467-1482, Mar. 2014, https://doi.org/10.3390/en7031467.

[2] D. N. Huu and V. N. Ngoc, "Analysis Study of Current Transportation Status in Vietnam's Urban Traffic and the Transition to Electric TwoWheelers Mobility," Sustainability, vol. 13, no. 10, Jan. 2021, Art. no. 5577, https://doi.org/10.3390/su13105577.

[3] D. N. Huu and V. N. Ngoc, "A Research on the Trend of Transport Electrification in Vietnam and the Feasibility of PV-Integrated Charging Station for Electric Two-wheelers at Electric Power University," in 11th International Conference on Power, Energy and Electrical Engineering, Shiga, Japan, Feb. 2021, pp. 255-260, https://doi.org/10.1109/ CPEEE51686.2021.9383333.

[4] E. Riva Sanseverino, H. Le Thi Thuy, M.-H. Pham, M. L. Di Silvestre, N. Nguyen Quang, and S. Favuzza, "Review of Potential and Actual Penetration of Solar Power in Vietnam," Energies, vol. 13, no. 10, Jan. 2020, Art. no. 2529, https://doi.org/10.3390/en13102529.

[5] M. Oakley, T. Dobson, H. Pham, and D. Tran, "Draft Power Development Planning 8 Of Vietnam," Energy Central, Aug. 26, 2021. https://energycentral.com/news/draft-power-development-planning-8vietnam (accessed Oct. 28, 2021)
[6] D. Fischer, A. Harbrecht, A. Surmann, and R. McKenna, "Electric vehicles' impacts on residential electric local profiles - A stochastic modelling approach considering socio-economic, behavioural and spatial factors," Applied Energy, vol. 233-234, pp. 644-658, Jan. 2019, https://doi.org/10.1016/j.apenergy.2018.10.010.

[7] K. Clement-Nyns, E. Haesen, and J. Driesen, "The Impact of Charging Plug-In Hybrid Electric Vehicles on a Residential Distribution Grid," IEEE Transactions on Power Systems, vol. 25, no. 1, pp. 371-380, Feb. 2010, https://doi.org/10.1109/TPWRS.2009.2036481.

[8] L. Pieltain Fernandez, T. Gomez San Roman, R. Cossent, C. Mateo Domingo, and P. Frias, "Assessment of the Impact of Plug-in Electric Vehicles on Distribution Networks," IEEE Transactions on Power Systems, vol. 26, no. 1, pp. 206-213, Feb. 2011, https://doi.org/10.1109/ TPWRS.2010.2049133.

[9] V. K. B. Ponnam and K. Swarnasri, "Multi-Objective Optimal Allocation of Electric Vehicle Charging Stations and Distributed Generators in Radial Distribution Systems using Metaheuristic Optimization Algorithms," Engineering, Technology \& Applied Science Research, vol. 10, no. 3, pp. 5837-5844, Jun. 2020, https://doi.org/ 10.48084/etasr.3517.

[10] M. E. Bendib and A. Mekias, "Solar Panel and Wireless Power Transmission System as a Smart Grid for Electric Vehicles," Engineering, Technology \& Applied Science Research, vol. 10, no. 3, pp. 5683-5688, Jun. 2020, https://doi.org/10.48084/etasr.3473.

[11] H. Aygun and M. Aktas, "A Novel DTC Method with Efficiency Improvement of IM for EV Applications," Engineering, Technology \& Applied Science Research, vol. 8, no. 5, pp. 3456-3462, Oct. 2018, https://doi.org/10.48084/etasr.2312.

[12] K. Zhang et al., "Optimal decentralized valley-filling charging strategy for electric vehicles," Energy Conversion and Management, vol. 78, pp. 537-550, Feb. 2014, https://doi.org/10.1016/j.enconman.2013.11.011.

[13] Z. Ma, D. Callaway, and I. Hiskens, "Decentralized charging control for large populations of plug-in electric vehicles: Application of the Nash certainty equivalence principle," in IEEE International Conference on Control Applications, Yokohama, Japan, Sep. 2010, pp. 191-195, https://doi.org/10.1109/CCA.2010.5611184.

[14] Z. Xu, Z. Hu, Y. Song, W. Zhao, and Y. Zhang, "Coordination of PEVs charging across multiple aggregators," Applied Energy, vol. 136, pp. 582-589, Dec. 2014, https://doi.org/10.1016/j.apenergy.2014.08.116.

[15] L. Jian, X. Zhu, Z. Shao, S. Niu, and C. C. Chan, "A scenario of vehicleto-grid implementation and its double-layer optimal charging strategy for minimizing load variance within regional smart grids," Energy Conversion and Management, vol. 78, pp. 508-517, Feb. 2014, https://doi.org/10.1016/j.enconman.2013.11.007.

[16] L. Zhang, F. Jabbari, T. Brown, and S. Samuelsen, "Coordinating plugin electric vehicle charging with electric grid: Valley filling and target load following," Journal of Power Sources, vol. 267, pp. 584-597, Dec. 2014, https://doi.org/10.1016/j.jpowsour.2014.04.078.

[17] N. Liu et al., "A Heuristic Operation Strategy for Commercial Building Microgrids Containing EVs and PV System," IEEE Transactions on Industrial Electronics, vol. 62, no. 4, pp. 2560-2570, Apr. 2015, https://doi.org/10.1109/TIE.2014.2364553.

[18] L. Jian, Y. Zheng, and Z. Shao, "High efficient valley-filling strategy for centralized coordinated charging of large-scale electric vehicles," Applied Energy, vol. 186, pp. 46-55, Jan. 2017, https://doi.org/ 10.1016/j.apenergy.2016.10.117.

[19] G. R. Chandra Mouli, P. Van Duijsen, F. Grazian, A. Jamodkar, P. Bauer, and O. Isabella, "Sustainable E-Bike Charging Station That Enables AC, DC and Wireless Charging from Solar Energy," Energies, vol. 13, no. 14, Jan. 2020, Art. no. 3549, https://doi.org/10.3390/ en13143549.

[20] D. K. Dhaked and D. Birla, "Microgrid Designing for Electrical TwoWheeler Charging Station Supported by Solar PV and Fuel Cell," Indian Journal of Science and Technology, vol. 14, no. 30, pp. 2517-2525, 2021.

[21] P. Fieltsch, H. Flamig, and K. Rosenberger, "Analysis of charging behavior when using battery electric vehicles in commercial transport," Transportation Research Procedia, vol. 46, pp. 181-188, Jan. 2020, https://doi.org/10.1016/j.trpro.2020.03.179. 
[22] Y. Zheng, Y. Shang, Z. Shao, and L. Jian, "A novel real-time scheduling strategy with near-linear complexity for integrating large-scale electric vehicles into smart grid," Applied Energy, vol. 217, pp. 1-13, May 2018, https://doi.org/10.1016/j.apenergy.2018.02.084.

[23] T. Mao, X. Zhang, and B. Zhou, "Intelligent Energy Management Algorithms for EV-charging Scheduling with Consideration of Multiple EV Charging Modes," Energies, vol. 12, no. 2, Jan. 2019, Art. no. 265, https://doi.org/10.3390/en12020265.

[24] C. Le Floch, F. Belletti, and S. Moura, "Optimal Charging of Electric Vehicles for Load Shaping: A Dual-Splitting Framework With Explicit Convergence Bounds," IEEE Transactions on Transportation Electrification, vol. 2, no. 2, pp. 190-199, Jun. 2016, https://doi.org/ 10.1109/TTE.2016.2531025.

[25] D. Nguyen Huu, "An Innovative Adaptive Droop Control Based on Available Energy for DC Micro Distribution Grids," Energies, vol. 13, no. 11, Jan. 2020, Art. no. 2983, https://doi.org/10.3390/en13112983.

[26] D. N. Huu, "An adaptive control of hybrid battery-supercapacitor storage for integration of wind and solar," in IEEE International Conference on Sustainable Energy Technologies, Hanoi, Vietnam, Nov. 2016, pp. 157 162, https://doi.org/10.1109/ICSET.2016.7811774.

[27] D. Nguyen Huu and H. T. Nam, "Adaptive coordinated droop control for multi-battery storage," in International Conference on Computer as a Tool, Salamanca, Spain, Sep. 2015, pp. 1-6, https://doi.org/ 10.1109/EUROCON.2015.7313703.

[28] K. Strunz, E. Abbasi, and D. N. Huu, "DC Microgrid for Wind and Solar Power Integration," IEEE Journal of Emerging and Selected Topics in Power Electronics, vol. 2, no. 1, pp. 115-126, Mar. 2014, https://doi.org/ 10.1109/JESTPE.2013.2294738. 